\title{
Development of Phase Contrast Scanning Transmission Electron Microscopy and its application
}

\author{
Hiroki Minoda $^{1 *}$, Takayuki Tamai ${ }^{1}$, Hirofumi Iijima ${ }^{2}$, Yukihito Kondo ${ }^{2}$ \\ ${ }^{1}$ Department of Applied Physics, Tokyo University of Agriculture and Technology, 2-24-16 Naka-cho, \\ Koganei, Tokyo 184-8588, Japan, \\ ${ }^{2}$ JEOL Ltd, 3-1-2 Musashino, Akishima, Tokyo 196-8558, Japan
}

An interaction between electron waves and biological molecules composed of light elements is very weak. This makes it difficult to obtain their high contrast images in transmission electron microscopy (TEM). A phase plate (PP) for TEM was realized by Danev and Nagayama in 2001[1] and contrast of the biological molecules can be enhanced. They made Zernike-type phase plate which is a thin carbon film and has a small hole in its center. It is placed at a back focal plane (BFP) of the objective lens (OL). It gives a phase shift of a half $\pi$ to scattering waves by means of the mean inner potential of the thin film and the unscattered wave was passed through the hole without giving a phase shift. Thus, the phase difference was provided among the scattering waves and un-scattering waves. It acts as a filter in the Fourier space and modifies the phase contrast transfer function (PCTF) of the OL form the sine-type to the cosine-type. Accordingly, phase contrasts of the biomolecules would be visualized.

There is a serious limitation in phase contrast TEM (P-TEM) because the PP system has to be installed in the BFP of the OL. The space round the BFP is limited and the PP can't be installed in commercial based high resolution TEM instruments because of their narrow gap of the OL pole piece. A special optical lens which can made a conjugate plane with the BFP of the OL is needed. To resolve this issue, we have been developing phase contrast transmission electron microscopy (P-STEM) by adjusting optical system including the PP system in the front focal plane (FFP) of the OL [2]. The PP for P-STEM is set in the condenser lens aperture plane (CLP) and the CLP was made to be the conjugate with the FFP by adjusting the optical illumination system.

We could demonstrate the possibility to realize the optical system of P-STEM. Change in the PCTF from the sine-type to the cosine-type cold be demonstrated, but the contrast enhancement of the biomolecules had not clearly demonstrated due to the electron damage. To resolve this issue intensity of the incident electron beam should be reduced. The reduced intensity of the incident beam decreases a signal to noise ratio. Moreover, we have to use a longer camera length in P-STEM than that in conventional-STEM (CSTEM) to reduce a background intensity that was under a C-STEM imaging condition as shown in Figure 1. The longer camera length reduces the P-STEM signal intensity. To improve the low signal level we have developed a new bright field detector for STEM. Figure 2 shows one of the examples comparing images of a hole of a carbon film. Both images were taken under the same condition. It clearly shows that an image obtained with the new detector in (b) is much brighter than that with the old one in (a). Intensity of the image at the vacuum region (inside the hole) in (b) is 3.6 times larger than that in (a). Wwe have been observe biological molecules and some of the samples can provide the enhanced phase contrast. We will introduce some application in our presentation.

[1] R. Danev and K. Nagayama, J. Phys. Sci. Jpn. 70 (2001), 696.

[2] H. Minoda, T. Tamai, H. Iijima, F. Hosokawa and Y. Kondo, to be published in Microscopy.

[3] This development was supported by SENTAN, JST. 


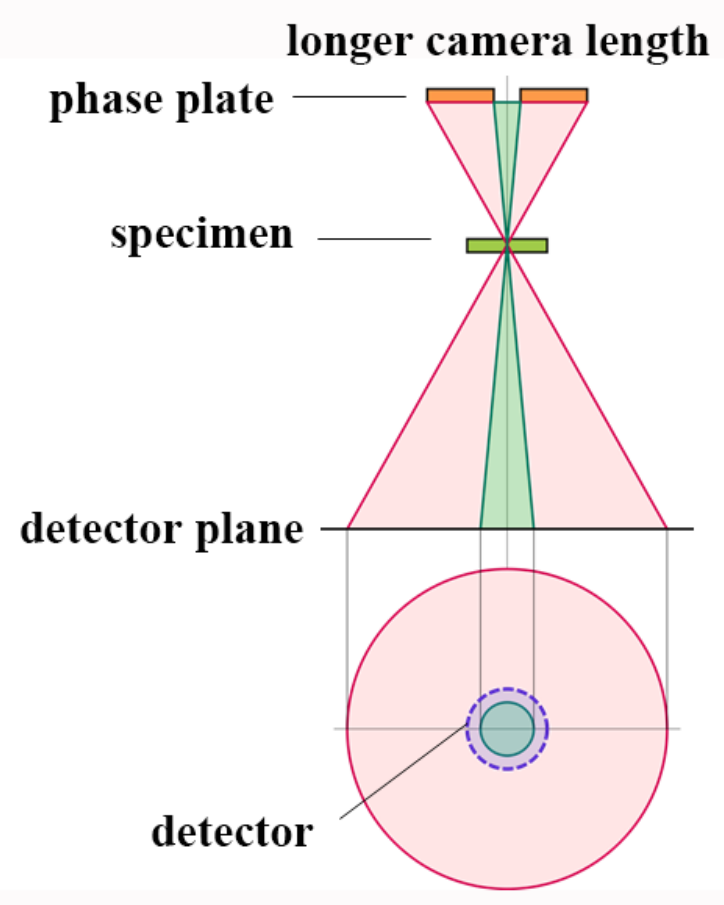

\section{shorter camera length}

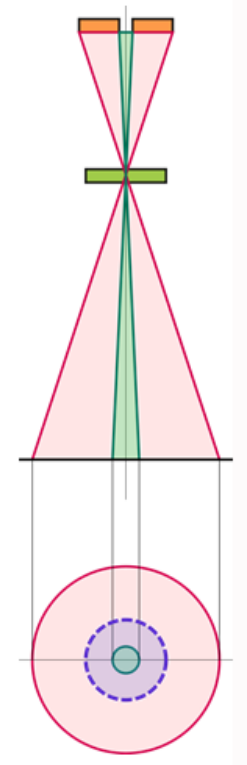

Figure 1 Projection image of the phase plate was formed on the detector plane and a camera length should be adjusted to fit the detector size to increase a signal to noise ratio.

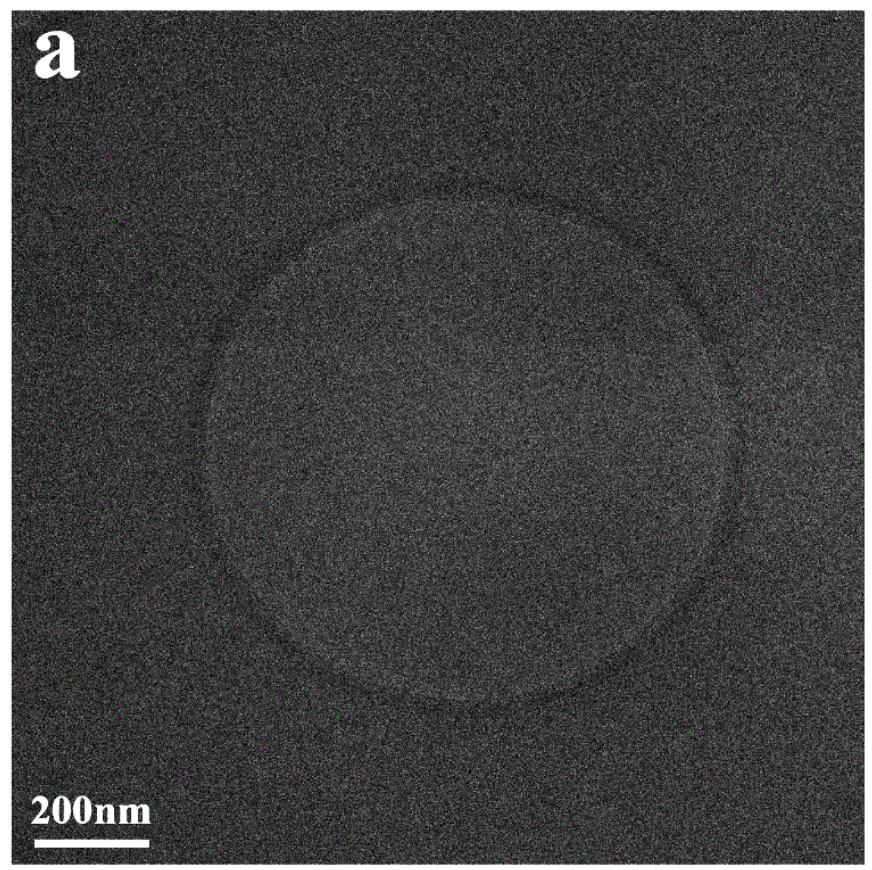

Figure 2 A comparison of the STEM bright field images obtained with an old (a) and a new detector. The image obtained by using the new detector is brighter than that obtained by the old detector. Signal intensity in (b) is about 3.6 times larger than that in (a). 